\title{
OPINION LETTER
}

\section{Mortalidad masiva de murciélagos en parques eólicos de España}

\author{
Comisión sobre murciélagos y parques eólicos ${ }^{1}$ \\ ${ }^{1}$ Asociación Española para la Conservación y el Estudio de los Murciélagos \\ *Corresponding author: eolica@secemu.org
}

DOI:https://doi.org/10.14709/BarbJ.14.1.2021.07

received: December, 1st 2020

accepted: May, 11th 2021

La adopción de actuaciones decididamente encaminadas a paliar y no agravar aún más las consecuencias del cambio climático en el que ya estamos inmersos es ineludible. En este sentido el posicionamiento de la SECEMU en relación con la necesidad de promover, desarrollar y optimizar sistemas de aprovechamiento energético sin emisiones asociadas es muy claro y absolutamente favorable. Sin embargo, los sistemas han de ser respetuosos con el medio ambiente que les rodea si pretenden ostentar el calificativo de "energía limpia". Y no se puede olvidar que la producción energética no es un servicio público, sino un negocio que mueve inversiones mil millonarias (se prevén en España entre 80 y 100 mil millones de euros en los próximos años, según la Asociación de Empresas de Energías Renovables EFE 2018) y genera importantes beneficios.

Los parques eólicos convencionales se han convertido ya en la primera causa de mortalidad de los murciélagos a nivel mundial (O’Shea et al. 2016), reconociéndose que el número de ejemplares muertos es además notablemente superior al de las aves (Smallwood 2013, Rydell et al. 2017, Thaxter et al. 2017). El problema amenaza ya el futuro de algunas especies (Frick et al. 2017). En España, hay 21419 turbinas en funcionamiento (27 $446 \mathrm{MW}$; AEE 2021). Las estimas realizadas a partir de datos de mortalidad recogidos en parques eólicos de la península ibérica (entre 3 y 10 murciélagos muertos por aerogenerador y año; DubourgSavage et al. 2011, Lekuona 2001, Camiña 2012, SánchezNavarro et al. in prep.) indican una media próxima a los 2 millones de murciélagos muertos en los últimos veinte años y evidencian la gravedad de la situación. En otros países europeos (Rydell et al. 2010, Georgiakakis et al. 2012, Voigt et al. 2015), Estados Unidos (Hayes 2013) o Canadá (Zimmerling \& Francis 2016), se han registrado tasas de mortalidad similares e incluso superiores. La preocupación por este elevado y continuado número de incidencias en un grupo de mamíferos que debido a su baja tasa de reproducción (generalmente una sola cría por hembra al año) se recupera muy mal ante pérdidas accidentales de sus poblaciones, ha motivado las alertas de la comunidad científica internacional (Kunz et al. 2007, Arnett et al. 2016)

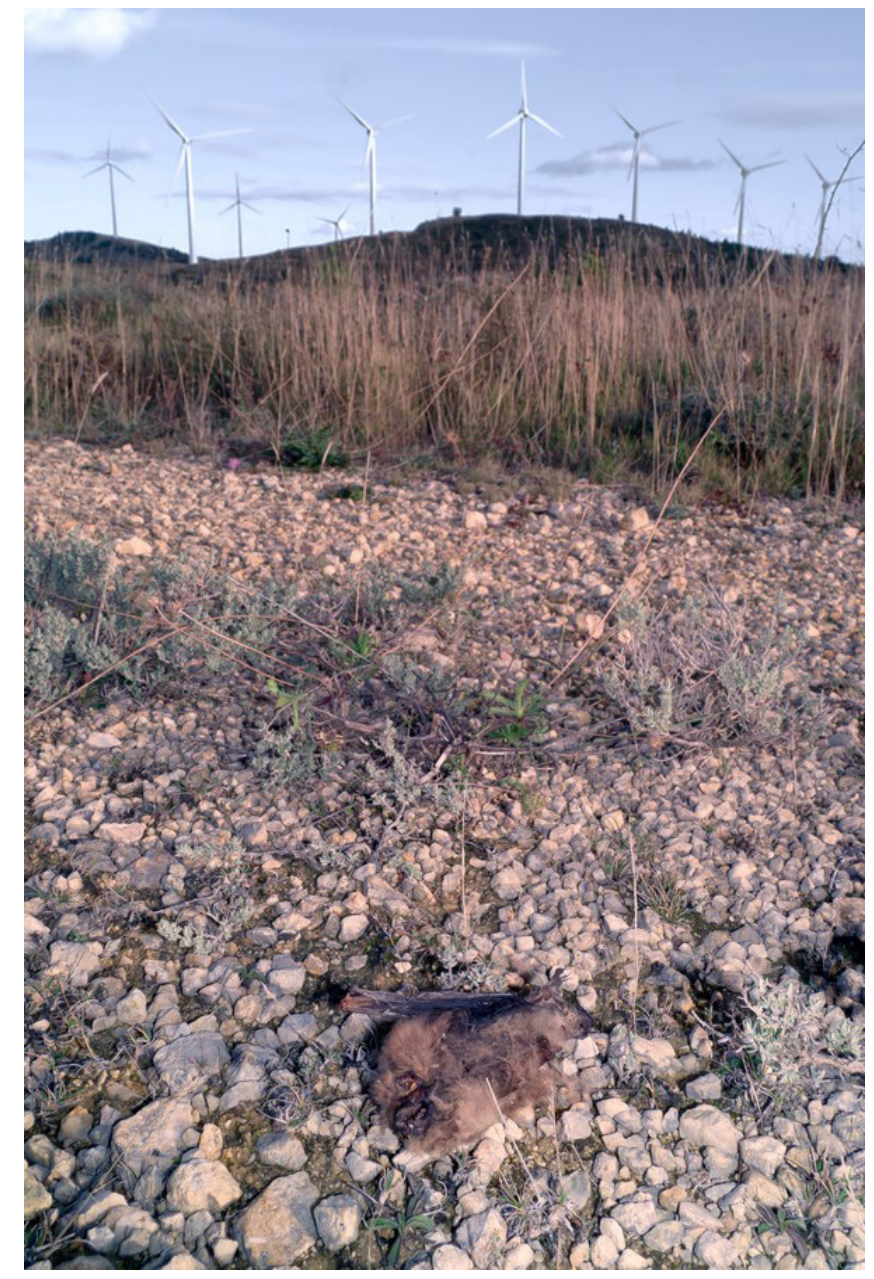

Fig. 1 - Eptesicus serotinus en un parque eólico del norte de España. Autor: Félix González.

y ha dado lugar a investigaciones para comprender los factores asociados a esta mortalidad (Barclay et al. 2007, Amorim et al. 2012, Cryan et al. 2014, Thompson et al. 2017) y minimizar el impacto (Baerwald et al. 2009, Arnett et al. 2011, Peste et al. 2015). 
La aplicación de los métodos de evaluación de impacto ambiental previa a la autorización de nuevos parques eólicos ha de ser, por tanto, particularmente rigurosa y se han elaborado directrices especiales para intentar evaluar correctamente el problema (en España ver González et al. 2013, en Europa ver Rodrigues et al. 2015). Sin embargo, en nuestro país este tipo de estudios consisten con demasiada frecuencia en un mero formalismo burocrático más, en el que la información aportada resulta manifiestamente deficiente e ineficaz para evaluar lo que se pretende (ver Camiña 2012). Además, se da la circunstancia de que quienes tienen que presentar este tipo de trabajos son directamente los propietarios de los propios parques eólicos, que son también los encargados del seguimiento de sus posibles efectos. Muchos de estos trabajos no reúnen una mínima calidad, se realizan sin el material de investigación adecuado o fuera de las fechas de actividad de los murciélagos (p. e., invierno) y aún así, suelen pasan los filtros de valoración de la Administración sin ningún tipo de problema. La misma Administración que ha de velar legalmente por la protección de los murciélagos (en España hay 34 especies, todas ellas protegidas por la ley; ver Real Decreto 139/2011). De hecho, suele ser habitual en estos trabajos la falta de cualquier referencia a la presencia de murciélagos, centrarse en solo una especie o basar las conclusiones en un número insignificante de observaciones. Todo ello cuando se trata del grupo de mamíferos con mayor número de especies en los ecosistemas terrestres españoles y que proporciona unos beneficios ecosistémicos fundamentales (ver por ejemplo, Boyles et al. 2011, Kunz et al. 2011, Ghanem \& Voigt 2012, Kasso \& Balakrishnan 2013). A pesar de vulnerar no pocas leyes relacionadas con la protección del medio ambiente (por ejemplo, Convenio de Berna, Convenio de Bonn, Directiva Hábitats, catálogos nacionales y autonómicos de especies amenazadas), la muerte de ejemplares no implica tampoco responsabilidad alguna y se intenta minimizar el problema evitando registrar la mortalidad o simplemente ignorándola. Considerando que los murciélagos son uno de los grupos de mamíferos más amenazados de nuestra fauna (Palomo et al. 2007) y el más afectado por el funcionamiento de los parques eólicos, es imprescindible adoptar mecanismos que minimicen realmente la sangría actual, empezando por mejorar los sistemas actuales de control, supervisión y evaluación utilizados por la Administración.

Con este panorama, el futuro próximo parece desolador. El Plan Nacional Integrado de Energía y Clima prevé pasar de los 28,033 MW eólicos estimados para 2020 a los 50,333 MW en 2030. Algunas actuaciones tan básicas como evitar el arranque de las máquinas cuando se registra la mayor mortalidad, a bajas velocidades de viento, pueden evitar en torno al $60 \%$ de las incidencias con menos del $1 \%$ de pérdida de la producción de energía (Lemaître et al. 2017). Sin embargo, de mantenerse la situación actual, sin adoptar ninguna medida, el impacto supondrá una mortalidad de otros 2 millones de murciélagos, en apenas diez años... Urgen actuaciones.

Algunas medidas prioritarias para para paliar el impacto de los parques eólicos sobre las poblaciones de murciélagos:

1. Seguimiento de protocolos específicos, claros y exigentes para la realización de estudios de impacto ambiental, Actualmente, el gobierno central y las comunidades de Navarra, Castilla y León, Peincipato de Asturias, Cataluña y la Rioja exigen ya protocolos específicos preoperacionales, aunque sus exigencias son diferentes.

2. Aplicación de medidas preventivas, correctoras y compensatorias específicas para las especies de murciélagos que sufren mortalidad en parques eólicos. Las medidas preventivas más efectivas son la instalación de parques en zonas con baja actividad de murciélagos y la elevación de la velocidad de arranque de los aerogeneradores a 5-6 m/s.

3. Aplicación de planes de vigilancia ambiental independientes de las empresas propietarias de los parques que hagan un seguimiento eficiente de la mortalidad, con test de detectabilidad y permanencia de cadáveres, plazos breves entre inspecciones (menos de 10 días) y uso de perros adiestrados para la búsqueda de cadáveres.

4. Cálculo de mortalidad estimada en cada parque, utilizando estos datos y aplicación de medidas correctoras si fuera preciso.

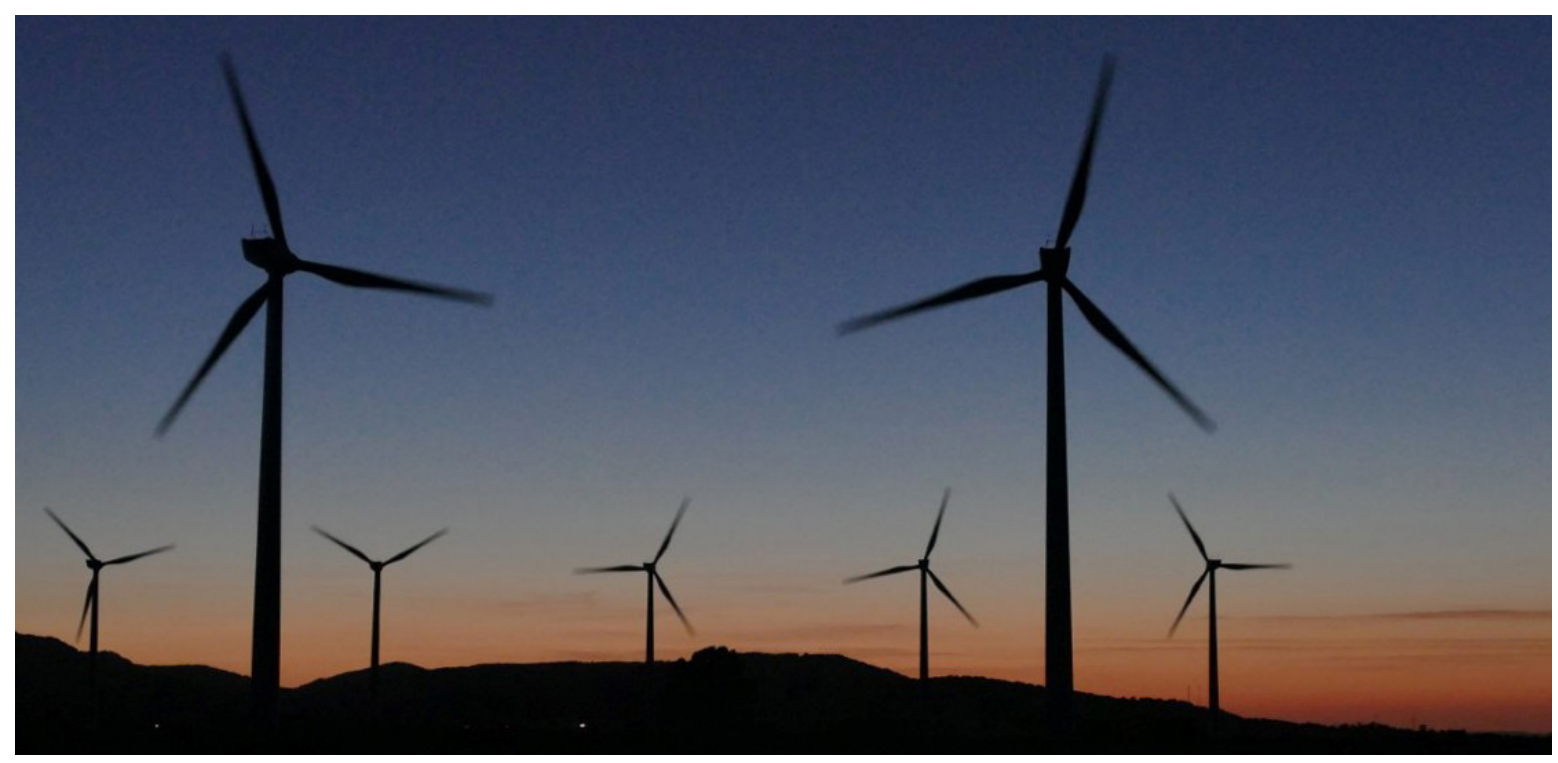

Fig. 2 - Parque eólico al atardecer. Autor: Juan Tomás Alcalde. 


\section{REFERENCIAS}

AEE (2021). Anuario eólico 2021. La voz del sector. Asociación Empresarial Eólica.

AMORIM, F., REBELO, H. \& RODRIGUES, L. (2012). Factors influencing bat activity and mortality at a wind farm in the Mediterranean region. Acta Chiropterol, 14(2): 439457. https://doi.org/10.3161/150811012X661756

ARNETT, E. B., BAERWALD, E. F., MATHEWS, F., RODRIGUES, L., RODRÍGUEZ-DURÁN, A., RYDELL, J., VILLEGASPATRACA, R. \& VOIGT, C. C. (2016). Impacts of Wind Energy Development on Bats: A Global Perspective. In: Bats in the Anthropocene: Conservation of Bats in a Changing World. ed.: Springer. Open eBook, p.295-323. https://doi.org/10.1007/978-3-319-25220-9_11

ARNETT, E. B., HUSO, M. M., SCHIRMACHER, M. R. \& HAYES, J. P. (2011). Altering turbine speed reduces bat mortality at wind-energy facilities. Front Ecol Environ, 9(4): 209214. https://doi.org/10.1890/100103

BAERWALD, E. F., EDWORTHY, J., HOLDER, M. \& BARCLAY, R. M. R. (2009). A Large-Scale Mitigation Experiment to Reduce Bat Fatalities at Wind Energy Facilities. J Wildlife Manage, 73(7): 1077-1081. https://doi. org/10.2193/2008-233

BARCLAY, R. M. R., BAERWALD, E. F. \& GRUVER, J. C. (2007). Variation in bat and bird fatalities at wind energy facilities: assessing the effects of rotor size and tower height. Can J Zool, 85(3): 381-387. https://doi.org/10.1139/Z07-011

BOYLES, J. G., CRYAN, P. M., MCCRACKEN, G. F. \& KUNZ, T. H. (2011). Economic Importance of Bats in Agriculture. Science, 332(6025): 41-42. https://doi.org/10.1126/ science. 1201366

CAMIÑA, A. (2012). Bat fatalities at wind farms in northern Spain - lessons to be learned. Acta Chiropterol, 14(1): 205-212. https://doi.org/10.3161/150811012X654402

CRYAN, P. M., GORRESEN, P. M., HEIN, C. D., SCHIRMACHER, M. R., DIEHL, R. H., HUSO, M. M., HAYMAN, D. T. S., FRICKER, P. D., BONACCORSO, F. J., JOHNSON,D. H., et al. (2014). Behavior of bats at wind turbines. P Natl Acad Sci USA, 111(42): 15126-15131. https://doi.org/10.1073/ pnas. 1406672111

DUBOURG-SAVAGE, M. J., RODRIGUES, L., SANTOS, H., GEORGIAKAKIS, P., PAPADATOU, E., BACH, L. \& RYDELL, J. (2011). Pattern of bat fatalities at wind turbines in Europe comparing north and south. Conference on Wind energy and Wildlife impacts, Trondheim, Norway, 2-5 May 2011.

EFE (2018). APPA dice que la cuota de renovables requerirá una inversión de 100.000 millones en España. El Diario.es, 14/06/2018. https://www.eldiario.es/economia/apparenovables-requerira-inversion-espana_1_2072284. html
FRICK, W. F., BAERWALD, E. F., POLLOCK, J. F., BARCLAY, R. M. R., SZYMANSKI, J. A., WELLER, T. J., RUSSELL, A. L., LOEB, S. C., MEDELLIN, R. A. \& MCGUIRE, L. P. (2017). Fatalities at wind turbines may threaten population viability of a migratory bat. Biol Conserv, 209: 172-177. https://doi. org/10.1016/j.biocon.2017.02.023

GHANEM, S. J. \& VOIGT, C. (2012). Chapter 7 - Increasing awareness of ecosystem services provided by bats. Adv Stud Behav, 44: 279-302. https://doi.org/10.1016/B9780-12-394288-3.00007-1

GEORGIAKAKIS, P., KRET, E., CÁRCAMO, B., DOUTAU, B., KAFKALETOU-DIEZ, A., VASILAKIS, D. \& PAPADATOU, E. (2012). Bat fatalities at wind farms in North-Eastern Greece. Acta Chiropterol, 14(2): 459-468. https://doi. org/10.3161/150811012X661765

GONZÁLEZ, F., ALCALDE, J. T. \& IBÁÑEZ, C. (2013). Directrices básicas para el estudio del impacto de instalaciones eólicas sobre poblaciones de murciélagos en España. SECEMU. Barbastella, 6 (núm. especial): 1-31.

HAYES, M. A. (2013). Bats killed in large numbers at United States wind energy facilities. BioScience, 63(12): 975979. https://doi.org/10.1525/bio.2013.63.12.10

KASSO, M. \& BALAKRISHNAN, M. (2013). Ecological and economic importance of bats (Order Chiroptera). Int Sch Res Notices, 187415: 9. https://doi. org/10.1155/2013/187415

KUNZ, T. H., ARNETT, E. B., ERICKSON, W. P., HOAR, A. R., JOHNSON, G. D., LARKIN, R. P., STRICKLAND, M. D., THRESHER, R. W. \& TUTTLE, M. D. (2007). Ecological impacts of wind energy development on bats: questions, research needs, and hypotheses. Front Ecol Environ, 5(6): 315-324. https://doi.org/10.1890/15409295(2007)5[315:EIOWED]2.0.CO;2

KUNZ, T. H., BRAUN DE TORREZ, E., BAUER, D., LOBOVA, T. \& FLEMING, T. H. (2011). Ecosystem services provided by bats. Ann NY Acad Sci, 1223(1): 1-38. https://doi. org/10.1111/j.1749-6632.2011.06004.x

LEKUONA, J. M. (2001). Uso del espacio por la avifauna y control de la mortalidad de aves y murciélagos en los parques eólicos de Navarra en un ciclo anual. Dirección General de Medio Ambiente, Gobierno de Navarra, Pamplona.

LEMAÎTRE, J., MACGREGOR, K., TESSIER, N., SIMARD, A., DESMEULES, J., POUSSART, C., DOMBROWSKI, P., DESROSIERS, N. \& DERY, S. (2017). Bat mortality caused by wind turbines: review of impacts and mitigation measures. ed.: Ministère des Forêts, de la Faune et des Parcs. Québec City, Canada, 26 pp.

O'SHEA, T. J., CRYAN, P. M., HAYMAN, D. T., PLOWRIGHT, R. K. \& STREICKER, D. G. (2016). Multiple mortality events in bats. Mammal Rev, 46(3): 175-190. https://doi. org/10.1111/mam.12064 
PALOMO, L. J., GISBERT, J. \& BLANCO, J. C. (2007). Atlas y Libro Rojo de los Mamíferos Terrestres de España. ed.: Dirección General para la Biodiversidad - SECEM SECEMU. Madrid, España, 588 pp.

PESTE, F., PAULA, A., DA SILVA, L. P., BERNARDINO, J., PEREIRA, P., MASCARENHAS, M., COSTA, H., VIEIRA, J., BASTOS, C., FONSECA, C., et al. (2015). How to mitigate impacts of wind farms on bats? A review of potential conservation measures in the European context. Environ Impact Asses, 51: 10-22. https://doi.org/10.1016/j. eiar.2014.11.001

RODRIGUES, L., BACH, L., DUBOURG-SAVAGE, M. -J., KAPARANDZA, B., KOVAC, D., KERVYN, T., DEKKER, J., KEPEL, A., BACH, P., COLLINS, J., et al. (2015). Guidelines for consideration of bats in wind farm projects. Revision 2014. ed.: UNEP/EUROBATS Secretariat. Bonn, Germany, 133 pp.

RYDELL, J., BACH, L., DUBOURG-SAVAGE, M. -J., GREEN, M., RODRIGUES, L. \& HEDENSTRÖM, A. (2010). Bat mortality at wind turbines in northwestern Europe. Acta Chiropterol, 12(2): 261-274. https://doi. org/10.3161/150811010X537846

RYDELL, J., OTTVALL, R., PETTERSSON, S. \& GREEN, M. (2017). The effects of wind power on birds and bats an updated synthesis report 2017. ed.: VINDVAL. The Swedish Environmental Protection Agency. Bromma, Sweden, $132 \mathrm{pp}$.
SÁNCHEZ-NAVARRO, S., RYDELL, J. \& IBÁÑEZ, C. (in prep.). High bat fatality rates estimated at wind farms in southern. Spain.

SMALLWOOD, K. S. (2013). Comparing bird and bat fatalityrate estimates among North American wind-energy projects. Wildlife Soc B, 37(1): 19-33. https://doi. org/10.1002/wsb.260

THAXTER, C. B., BUCHANAN, G. M., CARR, J., BUTCHART, S. H. M., NEWBOLD, T., GREEN, R. E., TOBIAS, J. A., FODEN, W. B., O'BRIEN, S. \& PEARCE-HIGGINS, J. W. (2017). Bird and bat species' global vulnerability to collision mortality at wind farms revealed through a trait-based assessment. P Roy Soc B-Biol Sci, 284(1862): 20170829. http://doi. org/10.1098/rspb.2017.0829

THOMPSON, M., BESTON, J. A., ETTERSON, M., DIFFENDORFER, J. E. \& LOSS, S. R. (2017). Factors associated with bat mortality at wind energy facilities in the United States. Biol Conserv, 215: 241-245. http://doi. org/10.1016/j.biocon.2017.09.014

VOIGT, C. C., LEHNERT, L. S., PETERSONS, G., ADORF, F. \& BACH, L. (2015). Wildlife and renewable energy: German politics cross migratory bats. Eur J Wildlife Res, 61: 213219. https://doi.org/10.1007/s10344-015-0903-y

ZIMMERLING, J. R. \& FRANCIS, C. M. (2016). Bat mortality due to wind turbines in Canada. J Wildlife Manage, 80(8): 1360-1369. https://doi.org/10.1002/jwmg.21128 